\title{
SINGULAR KOBAYASHI METRICS AND FINITE TYPE CONDITIONS
}

\author{
JIYE YU
}

(Communicated by Eric Bedford)

\begin{abstract}
We introduce a family of invariants associated with the higher order Kobayashi metrics and study their relations with the regular type in the sense of Kohn and the variety type in the sense of D'Angelo.
\end{abstract}

\section{INTRODUCTION}

The Kobayashi metric has proved to be very useful in studying many problems in complex analysis (cf., e.g., [KR1], [BP1], [BP2]). One of the most important topics has been the study of the relations between the optimal boundary asymptotic behavior of the metric on any weakly pseudoconvex domain and the boundary geometric invariants such as the types of the boundary. Though the problem is still open, there has been much encouraging progress (cf. [CA1], [CHE], [CHO], [DF], [HE], [YU], to name only a few). All the known results seem to suggest that the order of blow-up of the Kobayashi metric should have a tight relationship with the types of the boundary of the domain. One purpose of this paper is to show that the Kobayashi metric does measure the regular type (in the sense of Kohn) but not the variety type (in the sense of D'Angelo). In search of the appropriate substitute, we introduce a family of generalized Kobayashi metrics known as the higher order Kobayashi metrics and show that they measure the types precisely. Meanwhile, we found a new family of boundary invariants, namely, the k-type's which fit the Kobayashi metrics properly (for the definitions and precise statements, see the next section).

The author would like to express his deep gratitude to Professor Steven Krantz for his invaluable encouragement and inspiring suggestions. He would also like to thank Professor K. T. Kim and S. Fu for their interests in the subject.

\section{Definitions AND MAIN RESUltS}

Let $\Omega$ be a domain in $\mathbb{C}^{n}, p$ a smooth boundary point. We will denote by $H_{p}$ the complex tangent space of $\Omega$ at $p$. First we recall the definition of the

Received by the editors April 5, 1993; presented October 22, 1993 to the 886th AMS meeting, Texas A\&M University, College Station, Texas, in the Special Session on Several Complex Variables. 1991 Mathematics Subject Classification. Primary 32F15, 32H15.

Key words and phrases. Kobayashi metric, higher order Kobayashi metrics, singular Kobayashi metric, variety type, regular type. 
type which was introduced by D'Angelo [DA1] and we also define some related invariants.

Definition 1.1. Let $\rho$ be a local defining function for $\Omega$ near $p$. The variety type $\Delta_{1}(p)$ of $p$ (relative to $\Omega$ ) is defined as follows:

$$
\Delta_{1}(p)=\sup \left\{\frac{\nu(\rho \circ \varphi)}{\nu(\varphi)}: \varphi \in H\left(\Delta, \mathbb{C}^{n}\right) \backslash\{0\}, \varphi(0)=p\right\} .
$$

Here $\nu(f)$ denotes the order of vanishing of the mapping $f-f(0)$ at 0 . $H\left(\Delta, \mathbb{C}^{n}\right)$ denotes the space of analytic discs in $\mathbb{C}^{n}$. If we require that $\varphi^{\prime}(0) \neq 0$ in the definition of $\Delta_{1}(p)$, then we obtain the regular type of $p$, denoted by $\Delta_{1}^{r}(p)([\mathrm{KN}],[\mathrm{BG}])$.

We will also consider a slightly more general variety type called $k$-type ( $k$ is a positive integer), denoted by $\Delta_{1}(p, k)$, which is defined by

$$
\begin{aligned}
& \Delta_{1}(p, X, k)=\frac{1}{k} \sup \left\{\nu(\rho \circ \varphi): \varphi \in H\left(\Delta, \mathbb{C}^{n}\right),\right. \\
&\left.\varphi(0)=p, \nu(\varphi)=k, \varphi^{(k)}(0)=X\right\}, \\
& \Delta_{1}(p, k)=\sup \left\{\Delta_{1}(p, X, k): X \in H_{p}\right\} .
\end{aligned}
$$

Clearly we always have $\Delta_{1}^{r}(p)=\Delta_{1}(p, 1)$ and $\Delta_{1}(p)=\sup \left\{\Delta_{1}(p, k): k \geq 1\right\}$. Like the variety type, $k$-type does not depend on the local defining functions for $\Omega$ and is invariant under local holomorphic change of coordinates.

Next we define the higher-order Kobayashi metrics which are generalizations of the usual Kobayashi metric (for the properties of the Kobayashi metric, see [KB1], [RO]).

Definition 1.2. For any $z \in \Omega, X \in \mathbb{C}^{n}$, and integer $k \geq 1$, we define the $k$-th order Kobayashi metric by

$$
\begin{aligned}
& F_{\Omega}^{k}\left(z_{0}, X\right)=\inf \left\{\frac{1}{\lambda}: \varphi \in H(\Delta, \Omega) \text { with } \nu(\varphi-z)=k,\right. \\
& \left.\qquad \varphi^{(k)}(0)=k ! \lambda X, \lambda>0\right\} .
\end{aligned}
$$

The singular Kobayashi metric of $\Omega$ is defined by

$$
\hat{F}_{\Omega}(z, X)=\inf \left\{F_{\Omega}^{k}(z, X): k \geq 1\right\} .
$$

Here $H(\Delta, \Omega)$ denotes the set of complex discs in $\Omega$. Clearly $F_{\Omega}^{1}$ is just the standard Kobayashi metric which will be denoted by $F_{\Omega}^{K}$.

The higher order (and singular) Kobayashi metrics share many important properties with the standard Kobayashi metric, which we list in the following

Proposition 1.3. For each $k \geq 1$, denote by $F$ either $F_{\Omega}^{k}$ or $\hat{F}_{\Omega}$. We have:

(1) $F$ has the length decreasing property, i.e., if $f$ is a holomorphic map from a domain $D$ in $\mathbb{C}^{m}$ to another domain $\Omega$ in $\mathbb{C}^{n}$, then

$$
F_{\Omega}\left(f(z), f_{* z}(X)\right) \leq F_{D}(z, X), \quad \forall z \in D, X \in \mathbb{C}^{n} .
$$

In particular, $F$ is biholomorphically invariant.

(2) $F_{\Delta} \equiv F_{\Delta}^{K}$, the Kobayashi metric on the unit disc $\Delta$.

(3) $F_{\Omega}^{C}(z, X) \leq F_{\Omega}(z, X) \leq F_{\Omega}^{K}(z, X)$ for any domain $\Omega$ with $z \in \Omega$, $X \in \mathbb{C}^{n}$. Here $F_{\Omega}^{C}$ denotes the Carathéodory metric.

(4) $F_{\Omega}$ is continuous on the complex tangent bundle $T \Omega$ if $\Omega$ is taut. 
The proofs of these properties are straightforward and thus omitted (interested readers may consult [YU] for details).

Remarks. (i) There are many ways to construct higher order (or singular) invariant metrics, see [JP], [KB2], [WU] and references therein.

(ii) From Proposition 1.3, it is clear that the higher order (or singular) Kobayashi metrics can be estimated in a similar way as the standard Kobayashi metric, for further discussion please see [YU].

(iii) From (3) and the well-known fact ([LE], [RW]) that the Kobayashi metric and the Caratheodory metric coincide on bounded convex domains, we see that on any bounded convex domain $F_{\Omega}^{k}$ and $\hat{F}_{\Omega}$ coincide with the Kobayashi metric for any $k \geq 1$. On the other hand, the examples given in Section 3 will show that they are not equivalent in general.

Associated with any higher order Kobayashi metric, we can talk about its growth order which is defined as follows.

Definition 1.4. Let $\Omega, p, \rho$ be as in Definition 1.1. Let $\mathbf{n}$ be the unit outward normal vector at $p$ and $z_{t}=p-t$ n for $t>0$. Then, for any $k \geq 1, X \in H_{p}$, set

$$
\begin{aligned}
& t(p, X, k)=\underset{t \rightarrow 0^{+}}{\limsup } \frac{-\ln d_{\Omega}\left(z_{t}\right)}{\ln F_{\Omega}^{k}\left(z_{t}, X\right)}, \\
& t(p, k)=\sup \left\{t(p, X, k): X \in H_{p}\right\}, \quad \text { and } \\
& T(p)=\sup \{t(p, k): k \geq 1\} .
\end{aligned}
$$

Here $d_{\Omega}(z)$ denotes the Euclidean distance of $z$ from the boundary of $\Omega$. We call $t(p, k)$ (resp. $T(p))$ the growth order of the $k$-th order (resp. singular) Kobayashi metric. They measure how fast the metrics blow up near the boundary point. Observe that $t(p, k)$ and $T(p)$ are holomorphically invariant.

The first main result of this paper is:

Theorem 1. For any smooth boundary point $p$ of a domain $\Omega \subset \subset \mathbb{C}^{n}$, we have $\Delta_{1}(p, k) \leq t(p, k)$. In particular, the variety type $\Delta_{1}(p) \leq T(p)$ (the growth order of the singular Kobayashi metric).

A proof of this theorem (and a much stronger result) will be given in Section 2. Here we would like to draw a few interesting consequences from Theorem 1.

Corollary 1. Let $\Omega$ be a bounded domain in $\mathbb{C}^{n}$ and $p \in \partial \Omega$ a smooth boundary point. Then the regular type $\Delta_{1}^{r}(p)$ does not exceed the growth order of the Kobayashi metric.

In other words, the Kobayashi metric depends on the regular type. On the other hand, we will give examples (see Section 3) to show that it is independent of the variety type in general. However, for the Carathéodory metric, we have

Corollary 2. Let $\Omega$ be a bounded domain in $\mathbb{C}^{n}$ with a smooth boundary point $p$. If there exists a constant $m \geq 1$ such that for each $0 \neq X \in H_{p}$ one can find a constant $C(X)>0$ with

$$
F_{\Omega}^{C}\left(z^{j}, X\right) \geq C(X) d_{\Omega}\left(z^{j}\right)^{-1 / m}, \quad \text { for a nontangential sequence } z^{j} \rightarrow p,
$$

then $p$ is of finite type with $\Delta_{1}(p) \leq m$. 
This is a trivial consequence of the proof of Theorem 1 and the fact (3) of Proposition 1.3. It is also possible to prove this corollary directly by using the Cauchy estimates.

It was shown in [CA1] and [CHE] that if $\Omega \subset \subset \mathbb{C}^{2}$ is of finite type or $\Omega \subset \subset \mathbb{C}^{n}$ is convex and of finite type, then

$$
F_{\Omega}^{K}(z, X) \geq C\left|X_{T}(z)\right| d_{\Omega}(z)^{-1 / \Delta_{l}(p)} \quad \forall z \in \Omega \text { near } p \text { and } X \in \mathbb{C}^{n} .
$$

Here $p \in \partial \Omega$ and $C$ is a positive constant. $X_{T}(z)$ is the complex tangent component of the decomposition of $X$ at the nearest boundary point. However, such kind of sharp estimates cannot hold on any smooth domain in $\mathbb{C}^{n}$, because of the following

Corollary 3. Let $\Omega$ be a smooth domain in $\mathbb{C}^{n}$. If for any $p \in \partial \Omega$ there is a neighborhood $U$ of $p$ in $\mathbb{C}^{n}$ and a constant $C>0$ such that

$$
\hat{F}_{\Omega \cap U}(z, X) \geq C\left|X_{T}(z)\right| d_{\Omega}(z)^{-1 / \Delta_{1}(p)} \quad \forall z \in \Omega \cap U, X \in \mathbb{C}^{n},
$$

then the variety type $\Delta_{1}: \partial \Omega \cap U \rightarrow \mathbb{R}^{+}$is upper semi-continuous. Similarly, if we replace $\hat{F}_{\Omega \cap U}$ and $\Delta_{1}(p)$ by $F_{\Omega}^{K}$ and $\Delta_{1}^{r}(p)$ respectively in (1.2), then the regular type $\Delta_{1}^{r}$ is upper semi-continuous on $\partial \Omega \cap U$.

It is well known that the types are not upper semi-continuous on domains of finite type (cf. [DA1]), consequently, it is impossible to obtain an estimate analogous to (1.1) for all domains of finite type in $\mathbb{C}^{n}(n \geq 3)$. Corollary 3 can be verified readily from Theorem 1 and Corollary 1 .

It is natural to ask if the equality in Theorem 1 holds. In this regard we have the following result:

Theorem 2. If $\Omega \subset \subset \mathbb{C}^{2}$ or $\Omega \subset \subset \mathbb{C}^{n}$ (for any $n$ ) is locally convexifible at $p$, then $\Delta_{1}(p)=\Delta_{1}(p, k)=t(p, k)=T(p)$, provided $T(p)<\infty$.

The proof of Theorem 2 will also be given in Section 2. We remark that in case $\Omega$ is in $\mathbb{C}^{2}, \Delta_{1}(p)=t(p, 1)$ follows from the estimates obtained by Catlin [CA1].

Remarks. (i) Note that if $T(p)<\infty$ then the domain is necessarily pseudoconvex at $p$ ([YU]).

(ii) It follows from [GR] and Theorem 2 that a boundary point $p$ is strongly pseudoconvex if and only if $t(p, k)=T(p)=2$ for all $k$.

(iii) It follows from the work of [CHO] that $t(p, 1) \leq \Delta_{1}(p)^{N}$ for some large constant $N$. The sharp value of this $N$ is unknown.

(iv) Similar problems have also been studied recently by Diederich and Herbort [DH] for the invariants related to the Bergman metric as well as other analytic invariants.

\section{Proof of Theorem 1 and Theorem 2}

We first prove the following more general form of Theorem 1.

Theorem 3. Let $\Omega$ be a domain in $\mathbb{C}^{n}$ with a smooth boundary point $p$. Suppose that there exist constants $k \geq 1, m \geq 1$, a neighborhood $U$ of $p$, an $X \in H_{p}$, and a nontangential sequence $\left\{z^{j}\right\} \subset \Omega$ with $z^{j} \rightarrow p$ such that

$$
F_{\Omega \cap U}^{k}\left(z^{j}, X\right) \geq C(k, X) d_{\Omega}\left(z^{j}\right)^{-1 / m},
$$

for some positive constant $C(k, X)$. Then $\Delta_{1}(p, X, k) \leq m$. 
Proof. We may assume, without loss of generality, that $p=0$ and, near 0 , the domain $\Omega$ is defined by

$$
\rho(z)=2 \operatorname{Re} z_{n}+\mathscr{O}\left(|z|^{2}\right) .
$$

Assuming that $\Delta_{1}(0, X, k)>m$, we will seek a contradiction. By our hypothesis and definition, there exists a complex disc $\varphi$ in $\mathbb{C}^{n}$ such that $\varphi(0)=0$, $\nu(\varphi)=k, \varphi^{(k)}(0)=X$, and

$$
T=: \frac{1}{k} \nu(\rho \circ \varphi)>m+\varepsilon, \quad \text { for some sufficiently small } \varepsilon>0 .
$$

Here $T$ could be $\infty$ in which case we choose any finite $T>m+\varepsilon$. It follows that there is a constant $C_{0}>0$ such that

$$
|\rho(\varphi(\zeta))| \leq C_{0}|\zeta|^{k T}, \quad \text { for all small }|\zeta| .
$$

Now, let $z^{j}$ be given as in the theorem and put $\delta_{j}=d_{\Omega}\left(z^{j}\right)$. Define a family of complex discs by

$$
\varphi_{j}(\zeta)=\varphi\left(\delta_{j}^{\frac{1}{k(T-\varepsilon)}} \zeta\right)+z^{j} .
$$

Then we claim that $\varphi_{j}(\Delta) \subset \Omega \cap U$, if $j$ is sufficiently large. To this end, we first expand $\rho(z+w)$ at $z$ for $z, w$ near 0 :

$$
\rho(z+w)=\rho(z)+2 \operatorname{Re}\left(\sum_{i=1}^{n} \rho_{i}(z) w_{i}\right)+\mathscr{O}\left(|w|^{2}\right) .
$$

Here $\rho_{i}=\partial \rho / \partial z_{i}$ for $i=1,2, \ldots, n$ and $\mathscr{O}\left(|w|^{2}\right) \leq C|w|^{2}$ for some constant $C>0$ independent of $z$ and $w$. Note that $\left(\rho_{1}(0), \ldots, \rho_{n}(0)\right)=$ $(0, \ldots, 0,1)$ and by the continuity we see that for any small $\varepsilon_{0}>0$ there is a constant $r>0$ such that $|z|<r$ implies $\left|\rho_{i}(z)-\rho_{i}(0)\right|<\varepsilon_{0}$ for $i=1, \ldots, n$. It follows that, for all small $|z|,|w|$,

$$
\rho(z+w) \leq \rho(z)+2 \operatorname{Re} w_{n}+2 n \varepsilon_{0}|w|+C|w|^{2} .
$$

Next set $z=\varphi\left(\delta_{j}^{1 / k(T-\varepsilon)} \zeta\right)$ and $w=z^{j}=\left(z_{1}^{j}, \ldots, z_{n}^{j}\right)$ in (3). Since $z^{j} \rightarrow 0$ nontangentially, we may find positive constants $c_{1}, c_{2}$ such that for all $j$

$$
-\frac{1}{c_{1}} \delta_{j} \leq 2 \operatorname{Re} z_{n}^{j} \leq-c_{1} \delta_{j} \quad \text { and } \quad\left|z^{j}\right| \leq c_{2} \delta_{j}
$$

Moreover, since $\varphi(0)=0, \delta_{j} \rightarrow 0$, it is clear that $\left|\varphi\left(\delta_{j}^{1 / k(T-\varepsilon)} \zeta\right)\right| \leq r$ for all large $j$. Consequently for all $\zeta \in \Delta$ and sufficiently large $j$ we obtain, from (2)-(4), that

$$
\begin{aligned}
\rho\left(\varphi_{j}(\zeta)\right) & \leq \rho\left(\varphi\left(\delta_{j}^{\frac{1}{k(T-\varepsilon)}} \zeta\right)\right)+2 \operatorname{Re} z_{n}^{j}+2 n \varepsilon_{0}\left|z^{j}\right|+C\left|z^{j}\right|^{2} \\
& \leq C_{0}\left|\delta^{\frac{1}{(T-\varepsilon)}} \zeta\right|^{k T}-c_{1} \delta_{j}+2 n c_{2} \varepsilon_{0} \delta_{j}+c_{2} C \delta_{j}^{2} \\
& \leq C_{0} \delta^{\frac{T}{T-\varepsilon}}-\left(c_{1}-2 n c_{2} \varepsilon_{0}\right) \delta_{j}+c_{2} C \delta_{j}^{2} .
\end{aligned}
$$

Fix an $\varepsilon_{0}>0$ so small that $2 n c_{2} \varepsilon_{0} \leq c_{1} / 2$. Then it follows from (5) and $T /(T-\varepsilon)>1$ that $\rho\left(\varphi_{j}(\zeta)\right)<0$ for all $\zeta \in \Delta$ and sufficiently large $j$. This proves our claim that $\varphi_{j}(\Delta) \subset \Omega \cap U$ for all large $j$. 
Now it is easy to see from the definition of $\varphi_{j}$ that $\varphi_{j}(0)=z^{j}, \nu\left(\varphi_{j}\right)=k$, and

$$
\varphi_{j}^{(k)}(0)=\delta^{\frac{1}{T-\varepsilon}} \varphi^{(k)}(0)=\delta^{\frac{1}{T-\varepsilon}} X .
$$

Hence in view of the definition of $F_{\Omega}^{k}\left(z_{\delta}, X\right)$ we obtain

$$
F_{\Omega}^{k}\left(z_{\delta}, X\right) \leq \frac{1}{k !} \delta^{-\frac{1}{T-\varepsilon}}, \quad \text { for } j \text { sufficiently large. }
$$

However, by the hypothesis $(*)$, we have

$$
F_{\Omega}^{k}\left(z^{j}, X\right) \geq C(k, X) d_{\Omega}\left(z^{j}\right)^{-1 / m}=C(k, X) \delta_{j}^{-1 / m} .
$$

Thus we obtain from (6) and (7) that

$$
C(k, X) \delta_{j}^{-\frac{1}{m}} \leq \frac{1}{k !} \delta_{j}^{-\frac{1}{T-\varepsilon}}
$$

for all sufficient large $j$. This contradicts the assumption that $T>m+\varepsilon$ and $\delta_{j} \rightarrow 0$. This proves the first part of the theorem. The second part follows from the first part together with Definition 1.4.

Proof of Theorem 2. Note that $T(p)<\infty$ implies (by Theorem 1) that $\Delta_{1}(p)<$ $\infty$. Now we first consider the case $\Omega \subset \mathbb{C}^{2}$. In this case, it is well known that $\Delta_{1}^{r}(p)=\Delta_{1}(p)([\mathrm{DA} 1])$. We claim that $\Delta_{1}(p, k)=\Delta_{1}(p)$ for all $k \geq 1$. To see this, we may assume that $p=0$, and near $p, \partial \Omega$ is defined by a smooth real function $\rho$. Let $\Delta_{1}(p)=2 m$. After a standard change of coordinates, we may assume that [CA1]

$$
\rho(z)=\operatorname{Re} z_{2}+P\left(z_{1}\right)+\mathscr{O}\left(\left|z_{1}\right|^{2 m+1}+\left|z_{1}\right|\left|z_{2}\right|\right) .
$$

Here, we have written $z=\left(z_{1}, z_{2}\right)$, and $P$ is a real homogeneous subharmonic polynomial of degree $2 m$ which does not contain any harmonic terms. It is easy to see that, for the variety $\varphi_{0}(\zeta)=\left(\zeta^{k}, 0\right), \nu\left(\rho\left(\varphi_{0}\right)\right) \geq 2 m k$. Hence $\Delta_{1}(p, k) \geq 2 m$. Now suppose that $\Delta_{1}(p, k)>2 m$; we will derive a contradiction. It follows from the assumption and Definition 1.1 that there are constants $1>\varepsilon>0, C>0$, a variety $\varphi$ such that $\nu(\varphi)=k$, and

$$
|\rho(\varphi(\zeta))| \leq C|\zeta|^{2 m k+\varepsilon}, \quad \forall \zeta \in \Delta .
$$

We may write $\varphi(\zeta)=\left(\varphi_{1}(\zeta), \varphi_{2}(\zeta)\right)=\left(\zeta^{k} \psi_{1}(\zeta), \zeta^{k} \psi_{2}(\zeta)\right)$ for some holomorphic functions $\psi_{i}$ with $\psi(0)=\left(\psi_{1}(0), \psi_{2}(0)\right) \neq(0,0)$. In light of (8) and (9), we must have $\nu\left(\varphi_{2}\right) \geq 2 m k$. That is, $\left|\varphi_{2}(\zeta)\right| \leq C|\zeta|^{2 m k}$, for some constant $C>0$. Here and in the sequel we will use the same $C$ to denote possibly different constants. Therefore, it follows from (8), (9), and the above that, for all $\zeta \in \Delta$,

$$
\begin{aligned}
P(\varphi(\zeta)) & \leq C|\zeta|^{2 m k+\varepsilon}+C\left(|\zeta|^{2 m k+k}+|\zeta|^{k}|\zeta|^{2 m k}\right)-\operatorname{Re} \varphi_{2}(\zeta) \\
& \leq C|\zeta|^{2 m k+\varepsilon}-\operatorname{Re} \varphi_{2}(\zeta) .
\end{aligned}
$$

For any $r \in(0,1)$, by taking the integral along the circle $\{\zeta:|\zeta|=r\}$ and noting that $\operatorname{Re} \varphi_{2}$ is harmonic, we have

$$
\frac{1}{2 \pi} \int_{|\zeta|=r} P(\varphi(\zeta)) d \zeta \leq C r^{2 m k+\varepsilon} .
$$


The left-hand side of (11) is nonnegative by the subharmonicity and can be written as

$$
\frac{1}{2 \pi} \int_{0}^{2 \pi} P\left(r^{k} e^{i k \theta} \psi_{1}\left(r e^{i \theta}\right)\right) d \theta=\frac{1}{2 \pi} \int_{0}^{2 \pi} r^{2 m k} P\left(e^{i k \theta} \psi_{1}\left(r e^{i \theta}\right)\right) d \theta .
$$

This, together with (11), implies that for all $r \in(0,1)$,

$$
0 \leq \int_{0}^{2 \pi} P\left(e^{i k \theta} \psi_{1}\left(r e^{i \theta}\right)\right) d \theta \leq C r^{\varepsilon} .
$$

Letting $r \rightarrow 0^{+}$, we get $\int_{0}^{2 \pi} P\left(e^{i k \theta} \psi_{1}(0)\right) d \theta=0$. This is impossible since $\psi_{1}(0) \neq 0$ and $P$ is not harmonic. This contradiction proves our claim that $\Delta_{1}(p, k)=2 m$.

As we have already observed that $t(p, 1)=2 m$ is a consequence of [CA1]. For general $k \geq 1$, we use the estimates in [YU], namely,

$$
F_{\Omega}^{k}(z, X) \geq C|X| d(z)^{-1 / 2 m}, \quad \forall X \in H_{p}, \text { and } z \text { near } p .
$$

It follows immediately that $t(p, k) \leq 2 m$. On the other hand, by Proposition 1.3 , we always have $t(p, 1) \leq t(p, k)$ for any $k \geq 1$. Hence we obtain that

$$
2 m=t(p, 1) \leq t(p, k) \leq 2 m .
$$

This implies that $t(p, k)=2 m=\Delta_{1}(p, k)$, as desired.

In case $\Omega \in \mathbb{C}^{n}$ is locally convex near $p$, it is well known that $\Delta_{1}(p)=$ $\Delta_{1}^{r}(p)\left(=\Delta_{1}(p, 1)\right)$ ([MC]). By using special coordinates (cf. [CA2], [BP2], [MC]), one may assume that $\Omega$ is given near $p=0$ by $\rho(z)=\operatorname{Re} z_{1}+P\left(z^{\prime}\right)+$ $R(z)$, where $P$ is a weighted homogeneous plurisubharmonic polynomial with respect to the weight $\left(1 / m_{2}, \ldots, 1 / m_{n}\right)$ with $m_{2} \leq \cdots \leq m_{n}=\Delta_{1}(p)$ and $R(z)$ is the higher order remainder term. Observe that the convexity of $\Omega$ near $p$ implies that $P\left(z^{\prime}\right)>0$ except at $z^{\prime}=0$. Then following the same idea as above, one can easily prove that $\Delta_{1}(p, k)=\Delta_{1}(p)$ for any $k$. Thus to finish the proof, it remains to show that $t(p, k)=\Delta_{1}(p)$. To this end, we quote a result in [YU] as follows: in the special coordinates $\left(z_{1}, \ldots, z_{n}\right)$ as above, for any $k \geq 1$ and any nontangential sequence $\Omega \supset\left\{z_{j}\right\} \rightarrow p$, we have

$$
\begin{aligned}
C_{1}(k, X) & \leq F_{\Omega}^{k}\left(z_{j},\left(d X_{1}, d^{1 / m_{2}} X_{2}, \ldots, d^{1 / m_{n}} X_{n}\right)\right) \\
& \leq C_{2}(k, X), \quad \forall X \in \mathbb{C}^{n}, j \geq 1 .
\end{aligned}
$$

Here $C_{i}(k, X)>0$ are constants independent of $j$ and $d=d_{\Omega}\left(z_{j}\right)$. It follows immediately from this that $t(p, k)=m_{n}=\Delta_{1}(p)$.

Remark. As a matter of fact, Theorem 2 holds on a much larger class of domains called $h$-extendible domains than the class of finite type domains in $\mathbb{C}^{2}$ and finite type convexifible domains (see [YU] for details).

\section{EXAMPLES AND CONCLUDING REMARK}

In this section we discuss two examples to show that the standard Kobayashi metric does not depend on the variety type and the higher order Kobayashi metrics are not equivalent to each other.

Example 1. Consider the following pseudoconvex domain in $\mathbb{C}^{3}$ :

$$
D=\left\{\left(z_{1}, z_{2}, z_{3}\right): \rho=: 2 \operatorname{Re} z_{3}+\left|z_{1}^{2}-z_{2}^{3}\right|^{2}<0\right\}, \quad p=(0,0,0) .
$$


It is easy to see that $\Delta_{1}(p)=\infty$, while $\Delta_{1}^{r}(p)=6$ (see [DA2] or [KR2] for details). On the other hand, for any $X=(a, b, 0) \in H_{p}$ with $|a|^{2}+|b|^{2}=1$ and $z_{t}=(0,0,-t)$, we have the following estimates:

Claim. The Kobayashi metric for the domain $D$ at $z_{t}$ satisfies:

(i) $|a| t^{-1 / 4} \leq F_{D}\left(z_{t}, X\right) \leq t^{-1 / 4}$, if $a \neq 0$,

(ii) $F_{D}\left(z_{t}, X\right)=t^{-1 / 6}$, if $a=0$.

In particular, we have the estimate

$$
F_{D}\left(z_{t}, X\right) \geq C(X) d\left(z_{t}\right)^{-1 / 6}, \quad \forall X \in H_{p}, \text { where } C(X)= \begin{cases}|a||X|, & a \neq 0, \\ |X|, & a=0 .\end{cases}
$$

It follows that $t(p, 1)=\Delta_{1}^{r}(p)=6<\infty=\Delta_{1}(p)$.

The claim can be verified directly [YU].

This example also shows that the higher order Kobayashi metrics are not equivalent to the standard one. To see this, notice that by the claim, for the points $z_{t}=(0,0,-t)$ and the direction $X_{0}=(0,1,0)$, we have $F_{D}\left(z_{t}, X_{0}\right)=$ $t^{-1 / 6}$. On the other hand, since the discs $\varphi_{n}(\zeta)=\left(n^{3} \zeta^{3}, n^{2} \zeta^{2},-t\right)$ lie in $D$ for all $n \geq 1$, and $\varphi_{n}(0)=z_{t}, \varphi_{n}^{\prime}(0)=0, \varphi_{n}^{\prime \prime}(0)=2 n^{2} X_{0}$, it follows from the definition that $F_{D}^{2}\left(z_{t}, X_{0}\right) \leq 1 / n^{2}$ for all $n$. Therefore we must have $F_{D}^{2}\left(z_{t}, X_{0}\right)=0$. Thus $F_{D}^{2}$ cannot be equivalent to $F_{D}$.

The previous example can be modified slightly to give a more interesting one as follows.

Example 2. Consider the bounded pseudoconvex finite type domain:

$$
\tilde{D}=\left\{\left(z_{1}, z_{2}, z_{3}\right): \tilde{\rho}(z)=2 \operatorname{Re} z_{3}+\left|z_{1}^{2}-z_{2}^{3}\right|^{2}+\left|z_{1}\right|^{6}+\left|z_{3}\right|^{2}<0\right\} .
$$

Let $p=(0,0,0) \in b \tilde{D}$. It is easy to see that for all $m \geq 1: \Delta_{1}(p)=$ $\Delta_{1}(p, 2 m)=9$ but $\Delta_{1}^{r}(p)=\Delta_{1}(p, 2 m-1)=6$. Observe that $\rho(z) \leq \tilde{\rho}(z)$ for all $z \in \mathbb{C}^{n}$ (where $\rho$ is the defining function for $D$ given in Example 1), thus we have $\tilde{D} \subset D$. Moreover $D$ and $\tilde{D}$ have the same tangent space at $p$. Using the same idea of the computation in Example 1 we can easily obtain the following estimates for all $m \geq 1, z_{t}=(0,0,-t)$, and $X=(a, b, 0)$ :

$$
\begin{aligned}
F_{\tilde{D}}^{2 m-1}\left(z_{t}, X\right) & \geq C_{1}(X) d\left(z_{t}\right)^{-1 / 6}, \\
F_{\tilde{D}}^{2 m-1}\left(z_{t}, X\right) & \leq C_{2}(X) d\left(z_{t}\right)^{-1 / 6} \\
F_{\tilde{D}}^{2 m}\left(z_{t}, X\right) & \geq C_{3}(X) d\left(z_{t}\right)^{-1 / 9}, \\
F_{\tilde{D}}^{2 m}\left(z_{t}, X\right) & \leq C_{4}(X) d\left(z_{t}\right)^{-1 / 9} \quad \text { if } a=0, \\
& \text { if } a=0 .
\end{aligned}
$$

Here $C_{i}(X)>0$ are constants. Combining these estimates with Definition 1.4, we conclude that for the domain $\tilde{D}$ :

$$
\begin{aligned}
t(p, 2 m-1) & =\Delta_{1}(p, 2 m-1)=\Delta_{1}^{r}(p)=6, \\
t(p, 2 m) & =\Delta_{1}(p, 2 m)=\Delta_{1}(p)=9, \quad \forall m \geq 1, \\
T(p) & =\Delta_{1}(p)=9 .
\end{aligned}
$$

These assertions not only indicate that the standard Kobayashi metric cannot measure the variety type but also show that the singular Kobayashi metric is the right substitute. 
There is much evidence (cf. Theorem 2 and Example 2) to support our following conjecture:

For any smooth boundary point $p, \Delta_{1}(p, k)=t(p, k)$, for all $k \geq 1$. In particular, $\Delta_{1}(p)=T(p)$.

It seems to us (cf. the proof of Theorem 2) that in order to prove the conjecture, we need the sharp lower bounds for the higher order Kobayashi metrics on any finite type domains, which at the time of this writing are not yet available.

\section{REFERENCES}

[BG] T. Bloom and I. Graham, A geometric characterization of points of type $m$ on real submanifolds of $\mathbb{C}^{n}$, J. Differential Geom. 12 (1977), 171-182.

[BP1] E. Bedford and S. Pincuk, Domains with noncompact automorphism groups, J. Geom. Anal. 13 (1991).

[BP2] _ Convex domains with noncompact automorphism groups, Mat. Sb. (to appear).

[CA1] D. Catlin, Estimates of invariant metrics on pseudoconvex domains of dimension two, Math. Z. 200 (1989), 429-466.

[CA2] _ Boundary invariants of pseudoconvex domains, Ann. of Math. (2) 120 (1984), 529-586.

[CHE] J. Chen, Estimates of the invariant metrics on convex domains, Dissertation, Purdue Univ., 1989.

[CHO] S. Cho, A lower bound on the Kobayashi metric near a point of finite type, J. Geom. Anal. 2 (1992), 317-326.

[DA1] J. D'Angelo, Real hypersurfaces, order of contact, and applications, Ann. of Math. (2) 115 (1982), 615-637.

[DA2] _-, Several complex variables and geometry, CRC Press, Boca Raton, 1992.

[DF] K. Diederich and J. Fornæss, Proper holomorphic maps onto pseudoconvex domains with real analytic boundary, Ann. of Math. (2) 110 (1979), 575-592.

[DH] K. Diederich and G. Herbort, Geometric and analytic boundary invariants on pseudoconvex domains. Comparison results, preprint, 1993.

[GR] I. Graham, Boundary behavior of the Caratheodory metric and the Kobayashi metric on strongly pseudoconvex domains with smooth boundary, Trans. Amer. Math. Soc. 207 (1975), 219-240.

[HE] G. Herbort, Invariant metrics and peak functions on pseudoconvex domain of homogeneous finite diagonal type, Math. Z. 209 (1992), 223-243.

[JP] M. Jarnicki and P. Pflug, Invariant pseudodistances and pseudometrics-completeness and product property, Ann. Polon. Math. 55 (1991), 170-189.

[KB1] S. Kobayashi, Intrinsic distances, measures and geometric function theory, Bull. Amer. Math. Soc. 82 (1976), 357-416.

[KB2] _ A new invariant infinitesimal metric, Internat. J. Math 1 (1990), 83-90.

[KN] J. Kohn, Boundary behavior of $\bar{\partial}$ on weakly pseudoconvex manifolds of dimension two, J. Differential Geom. 6 (1972), 523-542.

[KR1] S. Krantz, Geometric analysis and function spaces, CBMS Regional Conf. Ser. in Math., No. 81, Amer. Math. Soc., Providence, RI, 1993.

[KR2] _ Function theory of several complex variables, 2nd ed., Wadsworth, Belmont, 1992.

[LE] L. Lempert, La metrique Kobayashi et las representation des domains sur la boule, Bull. Soc. Math. France 109 (1981), 427-474.

[MC] J. McNeal, Convex domains of finite type, J. Funct. Anal. 108 (1992), 361-373.

[RO] H. Royden, Remarks on the Kobayashi metric, Lecture Notes in Math., vol. 185, Springer, Berlin, 1971. 
[RW] H. Royden and P. Wong, Carathéodory and Kobayashi metric on convex domains, preprint, 1983.

[WU] $\mathrm{H} . \mathrm{Wu}$, Old and new invariant metrics on complex manifolds, Several Complex Variables: Proceedings of the Special Year held at the Mittag-Leffler Institute (Stockholm, 1987/1988) (J. Fornæss, ed.), Math. Notes, vol. 38, Princeton Univ. Press, Princeton, NJ, 1993.

[YU] J. Yu, Geometric analysis on weakly pseudoconvex domains, Dissertation, Washington University, 1993.

Mathematics Department, Washington University, St. Louis, Missouri 63130

Current address: Department of Mathematics, Texas A\&M University, College Station, Texas 77843-3368

E-mail address: jyu@math.tamu.edu 